\title{
THE CLINICAL CHARACTERISTICS OF SECONDARY HEADACHE
}

\author{
Shanmugasundaram $N^{1}$, Manickavasagam $J^{2}$, Gobinathan $S^{3}$, Balasubramanian $S^{4}$, Lakshmi Narasimhan $R^{5}$
}

${ }^{1}$ Assistant Professor, Institute of Neurology, Government Madras Medical College, Chennai.

${ }^{2}$ Assistant Professor, Institute of Neurology, Government Madras Medical College, Chennai.

${ }^{3}$ Professor, Institute of Neurology, Government Madras Medical College, Chennai.

${ }^{4}$ Professor, Institute of Neurology, Government Madras Medical College, Chennai.

5 Professor, Institute of Neurology, Government Madras Medical College, Chennai.

\section{ABSTRACT}

\section{BACKGROUND}

Given the range of disorders that are implicated in manifesting as headache, a detailed analysis and interpretation of the pattern of headache would be of immense help in distinguishing the rarer life-threatening causes of secondary headaches from the more common primary headaches.

\section{MATERIALS AND METHODS}

This is an observational study to analyse the headache pattern, secondary headache in patients having diagnosed to have brain tumour with the details of neoplasm by imaging and biopsy.

\section{RESULTS}

208 patients with brain neoplasm either primary or secondary were analysed. $11.5 \%$ did not have headache. During onset, supratentorial tumours had ipsilateral frontoparietal localisation, pituitary tumours and tumours causing raised intracranial pressure had bilateral and infratentorial tumours without raised ICP to occipital localisation. All these later had become generalised. More greater intensity headaches were seen with gliomas, meningioma, and tumours that have caused raised intracranial pressure. Intracranial pressure is more commonly seen with gliomas, ventricular tumours like ependymoma, colloid cyst of ventricles.

\section{CONCLUSION}

The features suggesting probable secondary cause are unilaterality, dull ache, recent onset or change in pattern with pre-existing headache, progressive in nature and in association with seizures or neurological deficits. The extent of tumour-associated oedema, and raised intracranial pressure are significantly important factors in tumour-associated headache.

\section{KEYWORDS}

Secondary Headache.

HOW TO CITE THIS ARTICLE: Shanmugasundaram N, Manickavasagam J2, Gobinathan S, et al. The clinical characteristics of secondary headache. J. Evolution Med. Dent. Sci. 2017;6(80):5632-5636, DOI: 10.14260/jemds/2017/1221

\section{BACKGROUND}

Given the range of disorders that are implicated in manifesting as headache, a detailed analysis and interpretation of the pattern of headache would be of immense help in distinguishing the rarer life-threatening causes of secondary headaches from the more common primary headaches.

\section{MATERIALS AND METHODS}

An observational study to analyse the headache pattern, secondary headache in patients having diagnosed to have brain tumour.

Patients in outpatient and inpatient departments of Neuromedicine and Neurosurgery in the Govt. hospital from Jan. 2016 to Dec. 2016.

'Financial or Other Competing Interest': None.

Submission 01-09-2017, Peer Review 23-09-2017,

Acceptance 30-09-2017, Published 05-10-2017.

Corresponding Author:

Manickavasagam. J,

Assistant Professor,

Institute of Neurology,

Government Madras Medical College,

Government General Hospital, Chennai.

E-mail:drjmanicks@gmail.com

DOI: $10.14260 /$ jemds/2017/1221

\section{Study Population}

Patients diagnosed to have primary or secondary brain tumour by neuroimaging.

\section{Exclusion Criteria}

Secondary headache due to Infections (e.g. abscess, encephalitis, meningitis, subdural empyema), Haemorrhage (intracerebral, subdural, subarachnoid), Arterial and venous thrombosis, Chiari malformations, extracranial and systemic causes.

All patients were interviewed about their personal details.

Any history of pre-existing headache, if change in pattern and quality of headache, current headache whether present or absent, if present, how long was the duration of the headache, Frequency - continuous, daily, $>15 /$ month, thrice weekly, twice weekly, once weekly, less than weekly), location (which side and whether frontal, temporal, parietal, occipital, hemispheric frontoparietal, to start with and during progression whether it becomes bilateral, generalised during the episode or does it remain strictly unilateral), intensity [on a nominal analogue scale (NAS) from 1 to 10 with 1 for little and 10 for worst pain], quality of pain (dull, stinging, pulsating, tightening or band like, other), duration of the event ( $<1$ hr., 1-4 hrs., 4-24 hrs., 24 hrs. to 3 days, 3-7 days, $>7$ days, no data); associated phenomenon during the 
episode-Nausea and vomiting. Photophobia, phonophobia, lacrimation,

Any worsening with coughing, exercise, bending down; any early morning worse headache with awakening; any features of raised intracranial pressure; any focal neurological findings associated since the onset of headache; any seizures associated since the onset of headache; whether needs medications for relieving headache.

The Nature of the Neoplasm was obtained (1) By Neuroimaging with regard to Tumour size was defined as the product of the two largest dimensions (in $\mathrm{cm}$ ) and was used as a measure to differentiate small $\left(<10 \mathrm{~cm}^{2}\right)$, medium (10$\left.20 \mathrm{~cm}^{2}\right)$ and large tumours $\left(>20 \mathrm{~cm}^{2}\right)$. Multifocal tumours were assigned to a special category. The surrounding oedema was estimated subjectively on a scale of $0-3$, from no oedema (0) to extensive oedema (3). The main location of the tumour was chosen from the following list: frontal, temporal, parietal, occipital, hemispheric-frontoparietal, temporoparietal or infratentorial, sellar, ventricular. (2) By histopathology,

The Statistical analysis was performed using SPSS package.

\section{RESULTS}

208 patients with brain neoplasm either primary or secondary were analysed of which $52.4 \%$ were male and $47.6 \%$ were female and the age ranged between 13 years to 85 years with mean age 40.99 .

Among these patients having brain neoplasm, 88.5\% ( $47.11 \%$ males and $46.35 \%$ females) had headache since the beginning or during the course of illness. $11.5 \%(5.29 \%$ males and $6.25 \%$ females) did not have headache during the course of illness till the time of interview.

The locations of tumours were frontal (21.6\%), parietal (22.1\%), temporal (5.1\%), occipital (1.4\%), CP angle (25\%), Ventricular (8.2\%), Sellar (5.8\%), Thalamic (1.4\%), Pineal (2.4\%), Multiple (1\%).71.2\% were in the supratentorial compartment and 28.8 were in the infratentorial compartment.

Of these, $27.9 \%$ were gliomas (including glioblastomas, astrocytomas, oligodendrogliomas), 26.4\% were meningiomas, $3.4 \%$ were pituitary macroadenomas, 0.5 craniopharyngiomas, $13.5 \%$ schwannomas more commonly in cerebellopontine angle, $3.8 \%$ were ependymomas, $9 \%$ were cysts (epidermoid, arachnoid and colloid cyst), 3.4\% were cerebellar haemangioblastomas, $1.9 \%$ were cavernomas, $4.8 \%$ were space-occupying lesions unclassified (biopsy not done), and 5.3\% were secondary metastatic from elsewhere in the body.

Gliomas (30.9\%) and meningiomas (29.8\%) were common in the headache group. In the no-headache group, $54.2 \%$ were $\mathrm{CP}$ angle vestibular schwannomas, $12.5 \%$ each were pituitary adenomas and secondary metastatic tumours, $4.2 \%$ each were of gliomas and cavernomas.

$1 \%$ had positive family history. 8 patients $(3.8 \%)$ had preexisting headache and had a change in some form of the pattern of headache. The frequency of episodes increased in 6 , remained the same in 2 , duration of episode was increased in 7 , remained the same in 1 . Headache onset location had changed in 1 , remained the same in 7 . All of them had noted change in the quality of headache in the recent past.The intensity increased in 2, remained the same in 6.
Almost all of 184 patients had an intermittent pattern of headache. In general, $15.2 \%$ had 8 episodes per month. $53.3 \%$ had frequency of 12 per month ( 3 episodes per week). $15.2 \%$ had 16 per month (4 days per week). $14.7 \%$ had daily headache. Most of the patients, $84.8 \%$ had headache duration ranging between 5 - 24 hours. $0.5 \%$ had headache duration less than 4 hours. $14.7 \%$ had headache duration more than 24 hours.

During onset, headaches were localised to frontoparietal side of tumour in supratentorial tumours, and bifrontoparietal in sellar tumours and in hydrocephalus cases, bi-occipital in infratentorial tumours. Regarding side of onset, $51.6 \%$ had onset in left side ipsilateral to the tumour, $27.2 \%$ had to right side ipsilateral to the tumour, $21.2 \%$ had bilateral headaches. $97.3 \%$ had progressed within half to one hour to become generalised. $2.7 \%$ had remained unilateral during the entire episode.

$97.3 \%$ reported that the headache was of dull aching quality and $2.7 \%$ had reported of having band like sensation over the head.

$49.5 \%$ had nominal analogue scaling intensity of $6.21 .2 \%$ had intensity of 5 and $19 \%$ had intensity scaling of 7 . Only $1 \%$ had intensity scaling of 8 .

$7.1 \%$ had intensity scaling of 4 and $1.9 \%$ had intensity scaling of 3 . In $31 \%$ of the patients, headache was associated with nausea and vomiting. 69\% did not have associated vomiting. $42.9 \%$ of patients reported that they had headache worsening during coughing. Only $10.3 \%$ had early morning headache while awakening from sleep. $12 \%$ had worsening of headache during exercise and $16.3 \%$ had worsening of headache during bending down.

$42.8 \%$ had features of raised intracranial tension. All the patients in the no-headache group with tumour did not have ICT.

About $38.5 \%$ had FND. In that $40.76 \%$ had headaches associated with focal neurological signs like hemiparesis, hemisensory impairment, ataxia, cranial nerve palsies, memory impairment, behaviour and personality change, etc.

$20.6 \%$ of 208 patients had seizure. In that $23.3 \%$ had headaches associated with seizures (Focal, focal with generalisation, generalised).

Isolated headache as the symptom was observed in $30 \%$ of the patients having headache. All patients (100\%) required headache relieving medication in the form of analgesics or in some patients, steroids for reducing tumour-associated oedema.

Regarding tumour size, $70.2 \%$ were small in size $(<10$ $\left.\mathrm{cm}^{2}\right) .28 .4 \%$ were of medium size $\left(10-20 \mathrm{~cm}^{2}\right), 1.4 \%$ were of large size $\left(>20 \mathrm{~cm}^{2}\right)$. There was no oedema in $61.1 \%$ of cases. $12 \%$ had mild oedema, $22.6 \%$ had moderate oedema, $4.3 \%$ had severe oedema.

\section{DISCUSSION}

Headache arises from activation of pain-sensitive intracranial structures. The intracranial pain-sensitive structures include the arteries of the circle of Willis and the first few centimetres of their medium-sized branches, meningeal (dural) arteries, large veins and dural venous sinuses, and portions of the dura near blood vessel including the tentorium and the diaphragma sellae.

Inflammation, traction, compression, malignant infiltration, and other disturbances of pain-sensitive 
structures lead to headache signals that reach the central nervous system (CNS) largely by way of the first division of the trigeminal nerve ( $\mathrm{CN} \mathrm{V}$ ), and therefore an occipital lobe tumour may refer pain to the frontal head region. Infratentorial lesions tend to refer pain posteriorly because this compartment is innervated by the second and third cervical nerve roots, which also supply the back of the head.

The prevalence of headache in the present study was 8.5\%. But previous studies have shown as follows: Forsyth and Posner[1]- 48\%, Pfund et al[2]- 58.8\%, Schankin et al[3]$60 \%$, Valentinis et al 2010[4]- $47.6 \%$. About $11.5 \%$ (5.29\% males and $6.25 \%$ females) did not have headache during the course of illness till the time of interview.

Regarding the type of tumours, gliomas (30.9\%) and meningiomas $(29.8 \%)$ were the most common in the headache group. In the no-headache group of patients, vestibular schwannomas (54.2\%) were most commonly seen in this study. But Pfund et al ${ }^{[2]}$ in their study had stated that in the headache group the most frequent findings were metastatic brain tumours and different astrocytomas. Hypophysis adenomas and glioblastoma multiforme were frequent in the no-headache group.

In ventricular tumours and in other infratemporal tumours with associated hydrocephalus and raised intracranial pressure, it was bifrontoparietal during onset. In infratentorial tumours, it was in occipital region during onset. These are due to region based stimulation and referred pain in the trigeminal nerve distribution in supratentorial or cervical nerve distribution in infratentorial tumours. These associations although corresponding to the location of tumour were not significant statistically (Left side $p=0.000$, bilateral $\mathrm{p} \quad$ value $=0.000, \quad$ right side $\mathrm{p}$ value $=0.417$ ) for all these locations of onset of headache. These observations are consistent with previous study. Forsyth and Posner et al ${ }^{[1]}$ who had stated that patients with unilateral headache had an ipsilateral tumour. Most patients with infratentorial tumours have supratentorial headaches only; but if occipital pain is present, an infratentorial tumour is more likely (Pfund et al[2]). Headache has lateralising value, especially in patients with supratentorial lesions who have no obvious increased intracranial pressure (Suwanwela et al[5]).

Infratentorial tumours were found to be significantly more often associated with occipital and less often with frontal headache. If headache was unilateral, then headache and tumour were on the same side in only $61.5 \%$ (Schankin et $\mathrm{al}^{[3]}$ ). But in contrast, other previous studies had stated headache lateralisation predicted tumour location in only one third of patients (Pfund et al 1999[2]). Frontal headaches were the most unreliable in predicting tumour location and were most common (Valentinis et al 2010[4]).

All the patients in the headache group had intermittent pattern of headache with varying frequency of episodes, most $(47.1 \%)$ of the patients had about 12 days a month and each episode lasting between 4-24 hours. This is consistent with

previous studies of Pfund et al[2] and Suwanwela et al[5] who had stated that the headache was never permanent and there was no regular daily recurrence.
More of supratentorial tumours had headache of relatively longer duration than infratentorial location. This was statistically significant $(\mathrm{p}=0.018)$.

In this study, $86.1 \%$ had dull aching type of pain and $2.7 \%$ had band like pain. This is consistent with Forsyth Posner,[1] Pfund et al,[2] Schankin et al[3] and Suwanwela et al. ${ }^{5}$

$49.5 \%$ had headache intensity under nominal analogue scaling of 6 and $19 \%$ had scaling of 7 and $21.2 \%$ had scaling of 5 .

$9.3 \%$ had scaling less than 5, suggesting that brain tumour-associated headache is a moderate to severe intensity type which is consistent with previous studies (Forsyth and Posner,[1] Pfund et al,[2] Schankin et al,[3] Valentinis et al.[4]) All the patients required analgesics for relieving their headache, some patients with tumourassociated oedema were benefitted from steroids.

$31 \%$ in the headache group had associated nausea and vomiting. $43 \%$ of the patients had headache worsening with cough, $16.3 \%$ had headache worsening with bending down, $12 \%$ had worsening during strain, $10.3 \%$ had early morning headache. $40.8 \%$ had focal neurological signs and $23.3 \%$ had seizures. These observations are consistent with previous studies that nausea and vomiting are reported in $18 \%$ to $60 \%$. And $20 \%$ reported headache exacerbation by Valsalva manoeuvres, about a third of patients have nocturnal or morning headaches or both. (Forsyth and Posner,[1] Pfund et al,[2] Schankin et al,[3] Valentinis et al.[4])

$42.8 \%$ had features of raised intracranial tension. All the patients in the no-headache group with tumour did not have raised intracranial pressure. This suggests that intracranial pressure is one of the important factor in the manifestation of headache. Among patients with raised intracranial pressure, $41.9 \%$ were of supratentorial and $45 \%$ were of infratentorial in location, implying that more of infratentorial tumours present with raised intracranial pressure. This association was not statistically significant ( $p$ value 0.4 ). Previous studies have stated that posterior fossa tumour causes headache more often than a supratentorial tumour (Purdy RA, Kirby S.[6]).

More of greater intensity headaches are seen with gliomas, meningioma, and tumours that have caused raised intracranial pressure. These are consistent with previous studies (Shankin et $\mathrm{al}^{[3]}$ ). With pituitary macroadenomas, both small and medium-sized tumours have caused moderate intensity headache. These observations are consistent with previous studies that pituitary volume and the presenting phenotype is likely to be governed by a combination of factors, including tumour activity, relationship to the cavernous sinus and patient predisposition to headache ( Levy et al[7]). 


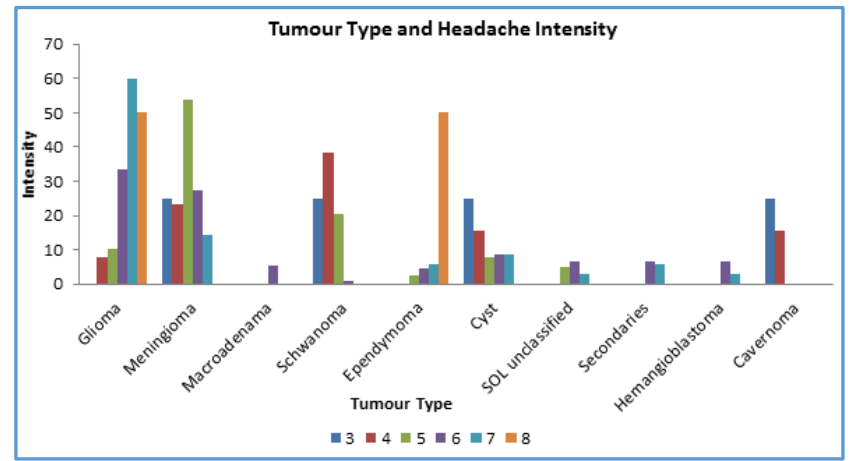

Figure 1. Headache Intensity (on a Nominal Analogue Scale (NAS) from 1 to 10) and Tumour Type

Most of medium and large size tumours have produced more intense headaches when compared with the small-sized tumours which have produced milder forms of headache. As certain small-sized tumours have also produced more intense headaches, probably some other factors like oedema may also be contributing to the intensity of the headache, suggesting that size of the tumour is one of the factor requiring modification by other factors like oedema, development of raised ICP, vascular or meningeal involvement, etc. in determining the headache intensity. The intensity of the headache has association with the size of the tumour which is significant $(p=0.000)$. Valentinis et al ${ }^{4]}$ have stated that within similar pathologies, increased size was associated with increased risk of headache, but other studies (Pfund et al 1999 [2]; Levy et al [7]; Schankin et al [3]) have not shown this association.

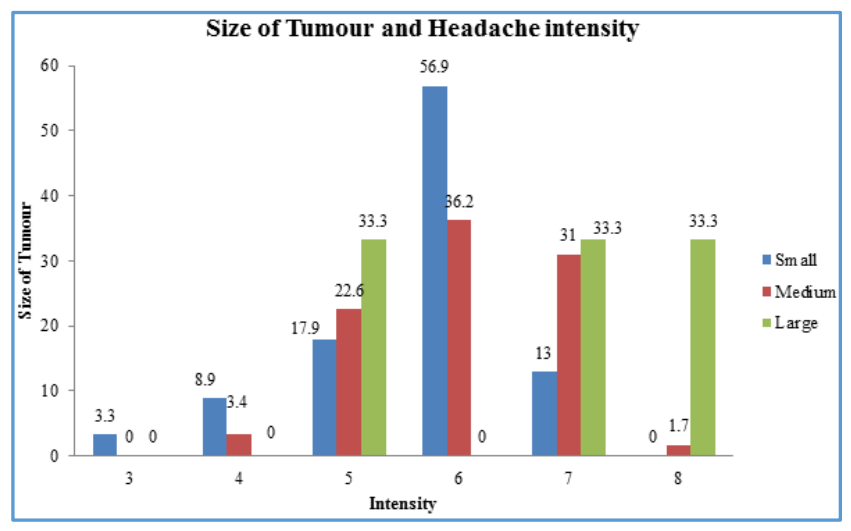

Figure 2. Headache Intensity (on a Nominal Analogue Scale (NAS) from 1 to 10) and Tumour Size

More number of patients with mild, moderate and severe oedema had more intense headaches than patients with no oedema. The intensity of the headache association with the extent of oedema due to tumour is significant $(\mathrm{p}=0.000)$.

Intense headaches of scaling 6 and more were seen in more number of patients with raised intracranial pressure group than in patients without raised intracranial pressure.

Intracranial pressure is more commonly seen with gliomas, in ventricular tumours like ependymomas, colloid cyst of ventricles. These associations are significant ( $\mathrm{p}$ value $=$ 0.000).

Even small-sized tumours with oedema have more intense headache than large tumours without oedema illustrating that oedema is an important factor in determining the intensity of the headache. The size of the tumour and oedema are important associated factors in determining the intensity along with other factors. Among them, oedema is more significant. This is statistically significant ( $p$ value 0.01 ).

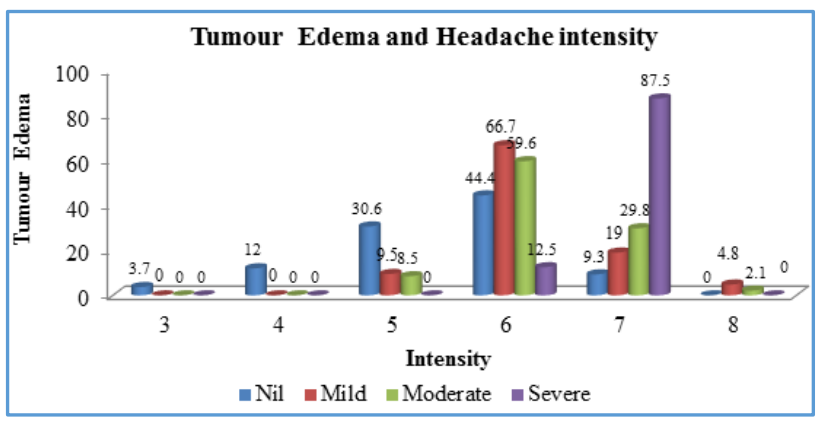

Figure 3. Headache Intensity (on a Nominal Analogue Scale (NAS) from 1 to 10) and Tumour Oedema (Nil, Mild, Moderate, Severe)

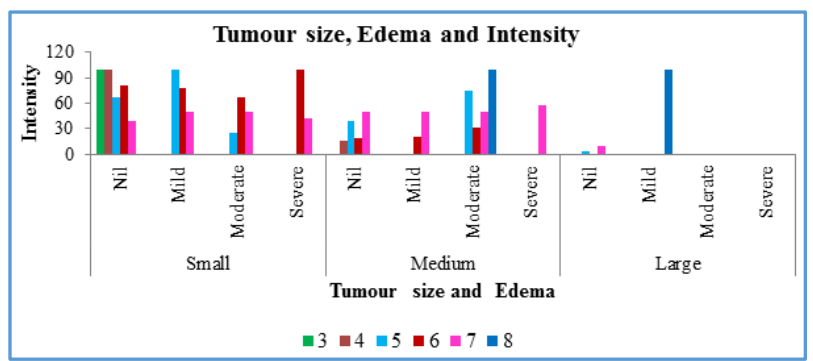

Figure 4. Headache Intensity (on a Nominal Analogue Scale (NAS) from 1 to 10) with Tumour Size \& Oedema (Nil, Mild, Moderate, Severe)

The frequency of episodes were less in the mild oedema group and more in the severe oedema group. The extent of oedema plays a significant role. This association is statistically significant ( $\mathrm{p}$ value 0.000 ).

Factors that predict increased risk of headache in patients with brain tumour include infratentorial location, raised intracranial pressure, degree of midline shift, and increasing oedema (Forsyth and Posner[1]; Pfund et al[2]). The relationship between tumour size and the likelihood of headache is uncertain. Similar to the findings of Forsyth and Posner, Valentinis et $\mathrm{al}^{4}$ found that within similar pathologies, increased size was associated with increased risk of headache, but others did not have similar findings (Pfund et al[2]; Levy et al[7]; Schankin et al[3]; Valentinis et al.[4]).

Even though only a small minority of patients with headache have brain tumour as the cause, it is crucial to recognise those headache characteristics that are associated with tumours. Because of the variable nature of headache among patients with brain tumour, the diagnosis of headache attributed to brain tumour should be considered in patients who complain of a headache with any of the following "red flags":

- Acute, new, usually severe headache or headache that has changed from previous patterns.

- New headache onset in an adult, especially over 50 years of age.

- Headache in the elderly or in children.

- Headache on exertion, onset at night, or onset at early morning. 
- Headache that is progressive in nature.

- Headache associated with fever or other systemic symptoms.

- Headache with meningismus.

- Headache with new neurologic signs.

- Precipitation of head pain with the Valsalva manoeuvre (by coughing, sneezing, or bending over).

In patients with any of the above features, further investigation with neuroimaging is warranted.

\section{CONCLUSION}

This study concludes that the clinical profile of brain tumourassociated headaches are having the special characteristic features of unilaterality, dull ache, recent onset or change in pattern with pre-existing headache, progressive in nature, headache intensity worsening with Valsalva, change in position or during exertion and in association with seizures or neurological deficits. The extent of tumour-associated oedema, and raised intracranial pressure are significantly important factors in tumour-associated headache.

Based on these special characteristic features, the study states that even if the headache does not have all the characteristic features, presence of any one or more of the abovementioned features should prompt suspicion of intracranial space-occupying lesion and should be investigated with neuroimaging for early diagnostic and therapeutic intervention.

\section{REFERENCES}

[1] Forsyth PA, Posner JB. Headaches in patients with brain tumors a study of 111 patients. Neurology 1993;43(9):1678-83.

[2] Pfund Z, Szapary L, Jaszberenyi O, et al. Headache in intracranial tumors. Cephalalgia 1999;19(9):787-90.

[3] Schankin CJ, Ferrari U, Reinisch VM, et al. Characteristics of brain tumour-associated headache. Cephalalgia 2007;27(8):904-11.

[4] Valentinis L, Tuniz F, Valent F, et al. Headache attributed to intracranial tumours a prospective cohort study. Cephalalgia 2010;30(4):389-98.

[5] Suwanwela N, Phanthumchinda K, Kaoropthum S. Headache in brain tumor: a cross-sectional study. Headache 1994;34(7):435-8.

[6] Kirby S, Purdy RA. Headache and brain tumors. Curr Neurol Neurosci Rep 2007;7(2):110-6.

[7] Levy MJ, Matharu MS, Meeran K, et al. The clinical characteristics of headache in patients with pituitary tumours. Brain 2005;128(Pt 8):1921-30. 